\title{
Outil de sensibilisation des proches à la conduite automobile des aînés*
}

\author{
Ariane Vigeant, ${ }^{2}$ Mylène Arseneault-Legault, ${ }^{2}$ Rachel Boily, ${ }^{2}$ Sarah Buchanan, ${ }^{2}$ \\ Jean-François Carosella, ${ }^{2}$ et Mélanie Levasseur ${ }^{1,2}$
}

\begin{abstract}
This study aimed to explore the impact of an awareness tool for relatives of older drivers (OSCARPA) on i) their interest, openness and knowledge, ii) changes of abilities required for safe driving, and iii) utilization of compensatory strategies. A pre-experimental design with pretest $\left(\mathrm{T}_{0}\right)$ and post-test $\left(\mathrm{T}_{1}\right)$ eight to ten weeks after the intervention was realized with 45 relatives in contact with an older driver of 65 years old or older and who was driving at least once a week. Overall, the results demonstrated that OSCARPA increased i) interest, openness and knowledge of relatives (p<0.001), as well as their perceptions of ii) changes of abilities of older drivers $(\mathrm{p}=0.02)$, and iii) their utilization of compensatory strategies $(p=0.001)$. Future studies would be relevant to further evaluate and increase the effectiveness of OSCARPA.
\end{abstract}

\section{RÉSUMÉ}

Cette étude visait à explorer les effets d'un outil de sensibilisation chez les proches de conducteurs âgés (OSCARPA) sur i) leur intérêt, ouverture et connaissances, ii) les changements des habiletés requises pour une conduite automobile sécuritaire, et iii) l'utilisation de stratégies compensatoires. Un dispositif pré-expérimental avec prétest $\left(\mathrm{T}_{0}\right)$ et post-test $\left(\mathrm{T}_{1}\right)$ huit à dix semaines après la remise de l'OSCARPA a été réalisé auprès de 45 proches côtoyant un conducteur âgé d'au moins 65 ans et conduisant au minimum 1 fois par semaine. Globalement, les résultats démontrent que l'OSCARPA augmente i) l'intérêt, l'ouverture et les connaissances des proches $(p<0,001)$, ainsi que leur perception ii) des changements de capacités du conducteur âgé $(p=0,02)$ et iii) de son utilisation de stratégies compensatoires $(p=0,001)$. Afin de poursuivre l'évaluation et d'accroître l'efficacité de l'OSCARPA, de futures études seraient pertinentes.

1 Centre de recherche sur le vieillissement, Sherbrooke, QC, Canada

2 Université de Sherbrooke, Faculté de médecine et des sciences de la santé, Sherbrooke, QC, Canada

* Nous tenons à remercier tous les participants de l'étude, Mme Lise Trottier, statisticienne du CdRV, Mme Joannie Collette, ergothérapeute, Pre Thérèse Audet, chercheure, Pre Isabelle Gélinas, ergothérapeute Ph.D., Pre Judith Renaud, Ph.D. optométrie, Dr Jamie Dow à la SAAQ, Mme Nathalie Drouin, conseillère en sécurité routière à la SAAQ et Mme Jade Bilodeau, adjointe administrative du CdRV. Lors de cette étude, Mélanie Levasseur était une chercheure boursière junior 1 du FRQS (\#26815) et elle est maintenant une nouvelle chercheure des IRSC (\#360880).

Manuscript received: / manuscrit reçu : 02/06/15

Manuscript accepted: / manuscrit accepté : 08/12/16

Mots clés : proche, sensibilisation, conducteur, aîné, OSCARPA, OSCAR

Keywords: relatives, awareness, driver, elderly, OSCARPA, OSCAR

La correspondance et les demandes de tire-à-part doivent être adressées à : / Correspondence and requests for offprints should be sent to:

Mélanie Levasseur, Ph.D

Université de Sherbrooke, Faculté de médecine et des sciences de la santé

3001, $12^{\mathrm{e}}$ avenue Nord

Sherbrooke, J1H 5N4 QC, Canada

(Melanie.Levasseur@USherbrooke.ca)

\section{Introduction}

Pour une proportion importante de la population, incluant les personnes âgées (Audet, Arcand, Godbout, et Lessard, 2007; Joseph, 2010), l'automobile est actuellement le principal moyen de transport. En 2009, 3,3 millions de personnes âgées de 65 ans et plus possédaient un permis de conduire valide, soit les trois quarts des Canadiens âgés de 65 ans et plus (Turcotte, 2012). Parmi ces conducteurs âgés, un nombre important présente une altération de leur santé susceptible d'affecter leur conduite automobile (Audet et al., 2007). De plus, le vieillissement normal peut aussi entraîner 
une détérioration des fonctions essentielles à une conduite automobile sécuritaire et responsable, c'est-àdire (c.-à-d). de la vision, de l'audition, du toucher, de la force musculaire, de la coordination, de la flexibilité, de l'attention et du jugement. Ainsi, les conducteurs âgés présentent un risque plus élevé d'avoir un accident de la route (Audet et al., 2007). Même lorsqu'ils ont conscience de leurs difficultés, la cessation de la conduite automobile est difficile pour les aînés, principalement en raison de son influence sur leur mobilité, leur participation sociale et leur qualité de vie (Classen et al., 2010; Eberhard et al., 2006). En effet, la conduite automobile est un symbole de fierté et de liberté, et permet aux conducteurs âgés d'être indépendants.

Afin de prolonger une conduite automobile sécuritaire, plusieurs conducteurs âgés ont besoin du soutien d'un professionnel de la santé ou d'un proche, et ce, surtout lorsqu'ils ont une perception altérée de leurs habiletés. Puisqu'ils les côtoient plus fréquemment que les professionnels de la santé, les proches ont un rôle clé à jouer auprès des conducteurs âgés (King, 2009). Le proche peut sensibiliser le conducteur âgé aux changements physiologiques qui surviennent avec l'âge et à leurs conséquences sur sa conduite automobile, ainsi que le renseigner sur les stratégies compensatoires à utiliser. Ces stratégies compensatoires, c.-à-d. les moyens utilisés par une personne pour pallier à la diminution de ses habiletés (Crepeau, Cohn, Schell \& Willard, 2009) qui inclut l'évitement de conduire le soir ou lors des heures de pointes et l'amélioration des habiletés de conduite via plusieurs cours / ressources disponibles, peuvent être discutées et expérimentées avec le soutien d'un proche. Ainsi, les proches doivent avoir une meilleure connaissance des habilités de conduite des aînés et de leur utilisation des stratégies compensatoires.

Toutefois, les discussions sur la conduite automobile entre le proche et le conducteur âgé sont rares et peuvent ne pas se dérouler tel que souhaité (King, 2009). La barrière la plus importante à ces discussions serait lorsque le proche ignore ses craintes envers la conduite automobile de l'aîné ( $\mathrm{D}^{\prime}$ Ambrosio et al., 2009). Par contre, le fait de planifier la discussion et d'envisager, dépendant de la situation, l'arrêt de conduire à court, moyen ou long terme (Liddle \& McKenna, 2003) serait un facilitateur majeur. Par ailleurs, puisque la majorité des conducteurs âgés ne prévoient pas modifier leur façon de conduire ou utiliser des stratégies compensatoires (Levasseur et al., 2014; Levasseur et al., 2015), les proches doivent être bien outillés pour mener ces discussions. Ainsi, afin de faciliter et de favoriser les discussions entre les proches et les aînés sur la conduite automobile (D'Ambrosio et al., 2009) et l'utilisation de stratégies compensatoires (Levasseur et al., 2015), il importe de sensibiliser et d'outiller les proches des conducteurs âgés.
Six outils identifiés dans les écrits scientifiques ont été développés en partie ou spécifiquement pour sensibiliser les proches de conducteurs âgés. Faciles à comprendre, ces outils sont tous disponibles en format papier et électronique (Tableau 1). Chacun de ces outils présente certaines caractéristiques particulières. Premièrement, l'outil 'We need to Talk: Family Conversations with Older Drivers' (Sedgwick County Department on Aging, 2010) présente les signaux d'alerte qui peuvent remettre en question une conduite sécuritaire chez l'aîné, par exemple, une diminution de la confiance lors de la conduite ou des difficultés pour se retourner lors de la marche arrière. Cet outil aide aussi le proche à identifier les alternatives à la conduite automobile, à évaluer la situation familiale et à orienter les discussions avec un aîné concernant la conduite automobile. Ensuite, la brochure 'Restez sur la bonne voie' (ACE, 2013) est un document de quatre pages, disponible en français, en anglais, en chinois, en italien et en pendjabi, qui présente une histoire de cas et soulève des questions sur les effets possibles du vieillissement sur la conduite automobile et les signes d'une conduite dangereuse. Cette brochure présente aussi quelques stratégies favorisant la sécurité au volant et pour la famille, aborde la cessation de conduire et identifie des ressources à consulter. Troisièmement, 1'outil 'Comment aider un aîné au volant pour des déplacements sécuritaires' (CAA, 2011) permet de prendre en compte la situation du proche et de l'aîné afin de favoriser une conduite automobile sécuritaire de l'aîné ou d'initier sa cessation de conduire. Quatrièmement, 1'outil 'Elderly Drivers: Is your loved one driving safely?' (Senior Solutions of America, 2007) présente une liste d'éléments à surveiller chez un aîné, ainsi que des ressources dont peuvent bénéficier les proches. Cinquièmement, l'outil 'Les conducteurs âgés au Canada et leur famille' (ACE, 2009) est un site web destiné aux conducteurs âgés et leur famille qui présente plusieurs conseils sur les signaux avertisseurs, les stratégies pour une conduite automobile sécuritaire, la mobilité dans la communauté et le renoncement à la conduite automobile. Enfin, la partie destinée aux proches de la brochure 'Au volant de ma santé' (SAAQ, 2011) expose différentes façons d'aborder la discussion avec l'aîné concernant l'arrêt de la conduite. Si l'aîné ne veut pas cesser de conduire, les deux alternatives suivantes sont suggérées : demander l'aide d'un établissement de santé et de services sociaux publique ou dénoncer l'aîné à la Société de l'assurance automobile du Québec (SAAQ). Offerte en français et en anglais et incluant une section pour les proches, la brochure est disponible aux points de services de la SAAQ et du Club de l'assurance automobile (CAA), et sur le site web de la SAAQ (SAAQ, 2011). De plus, six coordonnateurs régionaux de la SAAQ présentent les éléments clés de la brochure dans plusieurs regroupements d'aînés (FADOQ, cercle des fermières, etc.) à travers le Québec. 
Tableau 1 : Outils de sensibilisation destinés en tout ou en partie aux proches $(n=6)$

\begin{tabular}{|c|c|c|c|c|c|c|c|c|c|c|c|c|c|c|c|}
\hline Nom de l'outil & $\mathbf{i}$ & ii & iii & iv & $\mathbf{v}$ & vi & vii & viii & ix & $\mathbf{x}$ & xi & xii & xiii & xiv & Résultat total /13 \\
\hline $\begin{array}{l}\text { We need to Talk: Family Conversations with Older Drivers } \\
\text { (Sedgwick County Department on Aging, 2010) }\end{array}$ & & & & & $\checkmark$ & $\checkmark$ & & & & $\checkmark$ & $\checkmark$ & & $\checkmark$ & $\checkmark$ & 6 \\
\hline $\begin{array}{l}\text { Restez sur la bonne voie } \\
\text { (ACE, 2013) }\end{array}$ & & & & & $\checkmark$ & $\checkmark$ & & & & $\checkmark$ & & & $\checkmark$ & $\checkmark$ & 5 \\
\hline $\begin{array}{l}\text { Comment aider un aîné au volant pour des déplacements sécuritaires } \\
\text { (CAA, 2011) }\end{array}$ & & & & & $\checkmark$ & $\checkmark$ & & & & $\boldsymbol{V}$ & $\checkmark$ & & $\checkmark$ & & 5 \\
\hline $\begin{array}{l}\text { Elderly Drivers: Is your loved one driving safely? } \\
\text { (Senior Solutions of America, 2007) }\end{array}$ & & & & & $\checkmark$ & $\checkmark$ & $\checkmark$ & & & & & & $\checkmark$ & $\checkmark$ & 5 \\
\hline $\begin{array}{l}\text { Les conducteurs âgés au Canada et leur famille } \\
\text { (ACE, 2009) }\end{array}$ & & & & & $\checkmark$ & $\checkmark$ & & & & $\checkmark$ & & & $\checkmark$ & $\checkmark$ & 5 \\
\hline $\begin{array}{l}\text { Au volant de ma santé } \\
\text { (SAAQ, 2011) }\end{array}$ & & & & & $\checkmark$ & $\checkmark$ & & & & & & & & & 2 \\
\hline
\end{tabular}

\section{i - Fidèle}

ii - Valide (autre que de contenu)

iii - Sensible aux changements

iv - S'inspire d'un modèle théorique ou des données probantes

$\mathbf{v}$ - Disponible en version papier et électronique

vi - Facile à comprendre et à compléter (pas de comparaison sociale, langage simple et clair, absence de question à la négative)

vii - Temps requis pour compléter $(<15$ minutes $)$

viii - Permet la cotation numérique (score)

ix - Permet l'interprétation du score

$x$ - Divisé en sections pouvant être utilisées séparément

xi - Questions (énoncé nécessitant une réponse et qui peut amener à un score ou des capsules éducatives) plutôt qu'une liste de vérification (énoncé à cocher ou à lire, servant d'aide-mémoire ou de réflexion)

xii - Conseils personnalisés aux capacités de l'individu

xiii - Capsules éducatives expliquant le lien entre les déficiences et les incapacités associées à la conduite automobile

xiv - Propose des ressources supplémentaires 
Toutefois, à notre connaissance, ces six outils ne s'appuient pas sur de solides bases théoriques, n'ont pas été développés grâce à une synthèse rigoureuse des données probantes ou une consultation d'experts et n'ont pas été validés rigoureusement auprès de proches (Tableau 1). Par ailleurs, ces outils n'amènent pas directement le proche à se questionner sur les changements qui sont susceptibles d'être survenus chez le conducteur âgé à la suite du vieillissement, ni à les associer clairement aux stratégies à utiliser. De plus, aucune étude n'a démontré l'efficacité de ces outils. Ainsi, aucun de ces outils ne permet une sensibilisation optimale des proches à une conduite automobile sécuritaire et responsable des aînés. Par contre, un Outil de Sensibilisation aux habiletés requises et aux stratégies compensatoires pour une Conduite Automobile sécuritaire et Responsable (OSCAR), adapté à la population québécoise et disponible en français, permet d'augmenter l'intérêt, l'ouverture et les connaissances des aînés en lien avec le vieillissement et la conduite automobile (Levasseur et al., 2014; Levasseur et al., 2015). À l'aide d'une recension des écrits scientifiques et d'une consultation de six experts (trois intervenants dont deux ergothérapeutes et une optométriste, une chercheure et, provenant de la SAAQ, un médecin et une conseillère en sécurité routière, ayant une expertise en conduite automobile et vieillissement), l'OSCAR a été adapté à la réalité et aux responsabilités des proches de conducteur âgé (OSCARPA). L'adaptation de l'OSCARPA, comme le développement de l'OSCAR, a été guidée par plusieurs assises théoriques, soit : i) le Modèle du développement humain et du Processus de production du handicap (Fougeyrollas, 2010), explicatif du fonctionnement humain, ii) le modèle Transthéorique de Prochaska (Prochaska \& Velicer, 1997), descriptif de l'adoption de comportements, et iii) le modèle de Michon (1985), explicatif du traitement de l'information en conduite automobile. Cet outil représente un document de 25 pages incluant des informations utiles pour les proches de conducteurs âgés, notamment sur les effets du vieillissement sur les habiletés de conduite, les stratégies compensatoires et les ressources disponibles. L'OSCARPA inclut aussi des informations sur les facteurs qui influencent la discussion sur la conduite automobile, sur les processus de changement de comportement et sur un arbre décisionnel pour guider les actions des proches. Enfin, l'outil peut être accompagné d'un journal de bord qui permet aux proches de documenter ses stratégies et ses observations.

L'OSCARPA peut aider les différents professionnels de la santé à mieux conseiller les proches, augmenter leur ouverture à discuter de la conduite automobile et faciliter leurs interventions visant à assurer la sécurité du conducteur âgé. De plus, il permet de situer la conduite automobile en lien avec un continuum de ressources pour prolonger une conduite automobile sécuritaire des aînés et maintenir leur participation sociale. Cet outil peut aussi être utilisé en combinaison avec d'autres programmes, dont la plupart ont été développés pour les conducteurs âgés et non leur proche. Considérant que les proches auraient tendance à surestimer l'utilisation de stratégies compensatoires chez les conducteurs âgés (Levasseur et al., 2016), l'OSCARPA permet aussi de mieux les sensibiliser à l'importance de cette observation. Enfin, pour faciliter davantage les échanges entre un conducteur âgé et son proche, l'OSCAR et l'OSCARPA pourraient leur être respectivement remis. Avant de diffuser cette nouvelle intervention, les effets de l'OSCARPA auprès des proches doivent toutefois être vérifiés.

Cette étude visait donc à explorer les effets de l'OSCARPA sur i) l'intérêt, l'ouverture et les connaissances des proches, et leur perception ii) les changements des habiletés d'un conducteur âgé et iii) l'utilisation des stratégies compensatoires. Il est attendu, qu'à la suite de la lecture de l'OSCARPA et de l'utilisation du journal de bord, l'intérêt des proches participants, leur ouverture et leurs connaissances sur les habiletés nécessaires et les stratégies compensatoires pour une conduite automobile sécuritaire augmenteront. Par ailleurs, ils présenteront une prise de conscience accrue des changements en lien avec les habiletés de conduite d'un aîné et son utilisation des stratégies compensatoires.

\section{Méthodologie}

Dispositif de recherche

Afin d'évaluer les effets de 1'OSCARPA, un dispositif pré-expérimental incluant un prétest $\left(\mathrm{T}_{0}\right)$ et un post-test $\left(T_{1}\right)$ huit à dix semaines après l'intervention a été utilisé. Un délai de huit à dix semaines permettait aux proches de rencontrer à plus d'une reprise le conducteur âgé.

\section{Participants}

Grâce à une stratégie boule-de-neige (bouche-à-oreille) et à des affiches dans plusieurs lieux publics, 45 participants ont été recrutés. Les participants devaient communiquer en français et être le proche d'une personne âgée de 65 ans ou plus conduisant au moins une fois par semaine. Les personnes ayant accepté de participer étaient âgées entre 25 et 79 ans, la plupart était des femmes, avait plus de 14 ans de scolarité, travaillait à temps plein et était la fille ou le fils du conducteur âgé (Tableau 2).

\section{Procédure}

$\grave{A} \mathrm{~T}_{0}$, les participants étaient rencontrés individuellement pendant environ 60 minutes. Les questionnaires étaient d'abord complétés, puis les participants étaient 
Tableau 2 : Caractéristiques des participants $(n=45)$

\begin{tabular}{llc}
\hline \multicolumn{2}{l}{ Variable continue } & Moyenne (É.T) \\
\hline Âge & & $52,1 \pm 11,9$ \\
Variables catégoriques & $\mathbf{n}(\%)$ \\
Genre (Femme) & & $30(66,7)$ \\
Langue maternelle (Français) & $42(93,3)$ \\
Était civil & Marié/Conjoint & $34(75,6)$ \\
& Célibataire & $8(17,8)$ \\
Scolarité & Divorcé & $3(6,7)$ \\
& 11 ans et moins & $12(26,7)$ \\
& 12 à 14 ans & $11(24,4)$ \\
Occupation & Plus de 14 ans & $22(48,9)$ \\
& Travail temps complet & $28(62,2)$ \\
& Travail temps partiel & $3(6,7)$ \\
& Sans emploi & $1(2,2)$ \\
Lien avec le & Retraité & $12(26,7)$ \\
conducteur & Étudiant & $1(2,2)$ \\
âgé & Fille & $14(31,1)$ \\
& Fils & $12(26,7)$ \\
& Conjointe & $9(20,0)$ \\
& Conjoint & $2(4,4)$ \\
& Autre & $8(17,8)$ \\
\hline
\end{tabular}

invités à prendre connaissance de l'OSCARPA et ensuite du journal de bord (voir ci-dessous). Entre les deux temps de mesure, les participants conservaient l'OSCARPA et complétaient le journal de bord. À $\mathrm{T}_{1}$, les participants étaient de nouveau rencontrés individuellement et les mêmes questionnaires étaient administrés. Aucun abandon n'est survenu entre les deux temps de mesure. L'étude a été approuvée par le Comité d'éthique de la recherche du Centre de recherche sur le vieillissement (CdRV) du Centre de santé et de services sociaux de l'Estrie-Centre hospitalier universitaire de Sherbrooke (2014-433).

\section{Intervention}

Tel que mentionné, l'OSCARPA a été adapté à partir de l'OSCAR (Levasseur et al., 2014; Levasseur et al., 2015) et représente un document de 25 pages incluant les facteurs qui peuvent influencer la conduite automobile comme les habiletés, telles que le jugement, la vision, la force et la flexibilité, et les habitudes de vie, par exemple, la consommation d'alcool et de médicaments et les habitudes de conduite. Les facilitateurs et les barrières à la discussion sur la conduite automobile sont également abordés, dont, par exemple, l'importance d'avoir une attitude respectueuse et d'éviter de croire qu'il n'y a pas de problème en ignorant les craintes ressenties (D'Ambrosio et al., 2009; Eby \& Molnar, 2010; Friedland \& Rudman, 2009; Kostyniuk \& Molnar, 2008). De plus, l'OSCARPA présente un arbre décisionnel pour aider les proches d'aînés à choisir les solutions à mettre en place, notamment en lien avec les stratégies compensatoires et les alternatives à la conduite automobile comme le transport en commun et la bicyclette.
Enfin, l'outil inclut aussi une section sur les étapes des changements de comportements, telles que décrites par Prochaska (pré-réflexion, réflexion, préparation, action, maintien, rechute et terminaison; Prochaska \& Velicer, 1997), permettant aux proches de mieux comprendre où le conducteur âgé se situe dans ce processus. L'identification de l'étape à laquelle se trouve l'aîné permet au proche de le guider, de le motiver et d'établir des objectifs réalistes à propos de la conduite automobile et de l'utilisation de stratégies.

\section{Questionnaires et journal de bord}

Un questionnaire sociodémographique a tout d'abord été complété pour collecter les caractéristiques des participants. Adaptés à partir des versions pour les conducteurs âgés (Levasseur et al., 2014; Levasseur et al., 2015), trois autres questionnaires ont ensuite permis de mesurer les effets de l'OSCARPA chez les proches : 1) Intérêt, Ouverture et Connaissances chez les Proches d'Aînés (IOCPA), 2) Changements des Habiletés pour la conduite Automobile perçus par les Proches d'Aînés (CHAPA) et 3) Utilisation de Stratégies Compensatoires perçue par les Proches d'Aînés (USCPA). Le questionnaire Intérêt, Ouverture et Connaissances chez les Proches d'Aînés (IOCPA) comporte 36 questions ayant de 3 à 5 choix de réponses possibles, et portant sur trois dimensions : i) Intérêt envers l'information sur la conduite automobile et l'ouverture du proche et des aînés à discuter de ses capacités de conduite et de l'utilisation de stratégies compensatoires (12 questions, ex. Êtes-vous intéressé(e) par des reportages télévisés portant sur les habiletés à conduire des personnes âgées?; score variant entre 0-3, total /32), ii) Connaissances sur la sécurité routière et les habiletés requises (12 questions, ex. Quelle est l'importance d'avoir une bonne vision périphérique pour conduire une automobile?; score variant entre $0-1$, total /12), iii) Connaissances sur les effets du vieillissement et les stratégies compensatoires (12 questions, ex. Quel est l'effet du vieillissement sur la sensibilité aux éblouissements?; score variant entre 0-1, total /12). Le score total de l'IOCPA varie de 0 à 56, un score élevé représentant un intérêt, une ouverture et des connaissances supérieurs. Pour des fins de comparaison, les scores des dimensions et total sont rapportés en pourcentages. L'IOCPA, tel que calculé avec les données de la présente étude, démontrent une bonne cohérence interne (alphas de Cronbach : 0,74 à 0,77$)$. Le questionnaire Changements des Habiletés pour la conduite Automobile perçus par les Proches d'Aînés (CHAPA) fait ressortir les perceptions des proches quant aux changements pour 11 habiletés d'un conducteur âgé. Enfin, le questionnaire Utilisation de Stratégies Compensatoires perçue par les Proches d'Aînés (USCPA) permet aux participants de rapporter leur perception de l'utilisation de 20 stratégies compensatoires par le conducteur âgé. 
Les choix de réponse sur les changements des habiletés (CHAPA) ou l'utilisation des stratégies (USCPA) sont : observés par le proche, rapportés par le conducteur âgé ou quelqu'un de son entourage, aucun changement ou stratégie non utilisée (respectivement pour le CHAPA et l'USCPA), ou le proche ne sait pas. Le score total du CHAPA et de l'USCPA représente les changements de perception du proche entre $T_{0}$ et $T_{1}$, et varie de -1 à 1 . Les changements négatifs (-1) témoignent une sensibilisation mitigée, par exemple, une réponse "rapporté » à $\mathrm{T}_{0}$ suivi d'un " je ne sais pas » à $\mathrm{T}_{1}$. À l'inverse, en présence d'une bonne sensibilisation (changements positifs; 1), le participant répondait, par exemple, « je ne sais pas » à $\mathrm{T}_{0}$, puis " observé » à $\mathrm{T}_{1}$. Une réponse neutre (0), c.-à-d. identique à $T_{0}$ et à $T_{1}$, souligne une absence de sensibilisation (Annexes 1 et 2). Ainsi, pour chacun des éléments des questionnaires, un changement positif des réponses entre $T_{0}$ et $T_{1}$ suggère que le participant s'intéresse ou connaît davantage les habiletés de conduite ou les stratégies utilisées par le conducteur âgé. À l'inverse, afin d'être conservateur, un changement négatif ou neutre suggère que le participant ne s'intéresse pas ou ne connaît pas ces habiletés ou ces stratégies. Développée avec le soutien d'une statisticienne, cette stratégie permet de s'assurer d'une bonne sensibilité au changement sans surestimer les effets de façon positive. Enfin, à la suite de l'administration des questionnaires et de la lecture de l'OSCARPA, un journal de bord était remis aux participants afin de leur permettre de consigner leurs commentaires et leurs actions en lien avec l'étude. Ce journal de bord permettait d'écrire, par exemple, les sujets des conversations avec le conducteur âgé et des observations réalisées lorsque l'aîné conduisait.

\section{Analyse statistique}

Les participants et leur contexte ont d'abord été décrits à l'aide de moyennes et d'écarts-types ou de fréquences et de pourcentages, selon le type de variables (continues ou catégorielles respectivement). Pour évaluer l'efficacité de l'OSCARPA, un test $t$ bilatéral pour échantillons appariés, avec un intervalle de confiance de $95 \%$, a permis de comparer les résultats obtenus à $\mathrm{T}_{0}$ et à $\mathrm{T}_{1}$ aux trois questionnaires (IOCPA, CHAPA et USCPA) pour les scores totaux et, de façon exploratoire, pour chacune des dimensions de l'IOCPA, du changements des habiletés (CHAPA) et de l'utilisation des stratégies compensatoires (USCPA). Une analyse de contenu thématique des journaux de bord a permis de classifier en détail le contenu de l'ensemble des réponses des participants pour ensuite en faire une synthèse. Des extraits saillants et représentatifs ont été sélectionnés pour appuyer cette synthèse. Les analyses quantitatives ont été effectuées avec le logiciel SPSS (version 18.0) et, puisqu'elles étaient relativement simples, c.-à-d. réalisées en lien avec les journaux de bords complétés par les participants plutôt que sur des verbatims plus complexes, Word a été utilisé pour réaliser les analyses qualitatives.

\section{Résultats}

Avant l'intervention, les participants ont obtenu un score moyen légèrement inférieur au $2 / 3$ des points à 1'IOCPA (Tableau 3). Un score inférieur a été obtenu à la dimension "Intérêt et ouverture ", alors que les "Connaissances sur la sécurité routière et les habiletés requises » était la dimension pour laquelle le score moyen était le meilleur. À la suite de la lecture de l'OSCARPA et de l'utilisation du journal de bord, l'intérêt, l'ouverture et les connaissances des proches ont augmenté (Tableau 3). Plus spécifiquement et toujours pour 1'IOCPA, les proches se sont principalement améliorés aux dimensions "Connaissances sur l'effet du vieillissement et les stratégies compensatoires » $(9,4 \%)$ et "Intérêt et ouverture » $(5,7 \%)$, mais moins pour les «Connaissances sur la sécurité routière et les habiletés requises » $(5 \%)$.

À la suite de la lecture de l'OSCARPA et de l'utilisation du journal de bord, les proches ont aussi amélioré leurs perceptions quant aux changements des habiletés du conducteur âgé (CHAPA; Tableau 4). La moyenne de chaque habileté indique, de façon exploratoire, une tendance vers une amélioration, mais ce changement est statistiquement significatif pour 2 des 11 habiletés, soit « Traiter les informations provenant de différentes sources » et "Rester attentif sur la route quand il ressent de la douleur».

De plus, à la suite de l'OSCARPA et de l'utilisation du journal de bord, les proches ont amélioré leurs perceptions quant à l'utilisation de stratégies compensatoires par le conducteur âgé (USCPA; Tableau 5). La moyenne de la majorité $(90 \%)$ des stratégies compensatoires indique, de façon exploratoire, une tendance vers une amélioration, mais ce changement est statistiquement significatif pour 5 des 20 stratégies compensatoires, soit «Regarder à droite de la chaussée plutôt que les phares des voitures venant en sens inverse ", "Être plus conscient de ce qui se passe tout autour de lui », "Porter une attention particulière à la circulation, aux panneaux de signalisation, aux feux de circulation, aux cyclistes et aux piétons à l'approche des intersections ", "Conduire à la limite de vitesse permise » et " $\hat{t}$ tre à l'écoute des commentaires de son entourage concernant sa conduite ». Par ailleurs, les perceptions en lien avec les deux stratégies compensatoires suivantes n'ont pas changé à la suite de l'OSCARPA : «Éviter de conduire à la noirceur » et "Éviter de conduire lors de conditions pouvant interférer avec l'habileté à se concentrer » (Tableau 5). Les connaissances des participants quant aux changements des habiletés du conducteur âgé ou à l'utilisation des stratégies ont aussi augmenté à la suite 
Tableau 3 : Résultats du questionnaire Intérêt, Ouverture et Connaissances des Proches d'Aînés (IOCPA; n = 45)

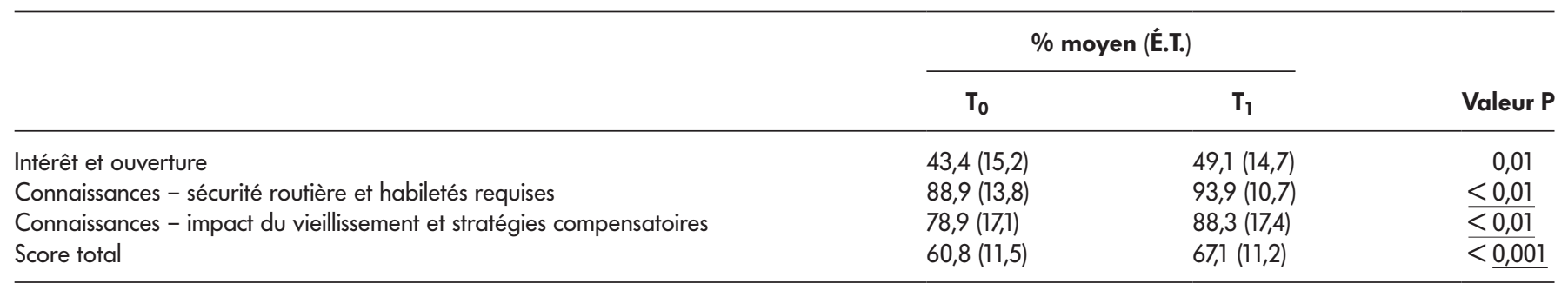

$T_{0}$ : prétest

$T_{1}$ : post-test

de l'OSCARPA (diminution des réponses "je ne sais pas » et augmentation des réponses " changement/ stratégie observé » et "changement/stratégie rapporté " des Annexes 3 et 4). Les participants ont également eu tendance à sous-estimer les changements dans les habiletés et l'utilisation des stratégies compensatoires par le participant (augmentation des réponses « aucun changement » de l'Annexe 3 et diminution des réponses "stratégie non utilisée » de l'Annexe 4).

Enfin, selon les journaux de bord, les participants ont rencontré approximativement 5 fois (médiane : 3 ; intervalle interquartile : 8) le conducteur âgé pendant la période de huit à dix semaines et l'ont vu conduire environ 3 fois (médiane :1; intervalle interquartile : 3 ). Toujours selon ces journaux, plusieurs proches ont apprécié l'OSCARPA, notamment puisqu'il les avait aidés à guider leurs observations en lien avec le niveau de sécurité du conducteur âgé, tel que le rapporte cette participante pour sa mère : "L'OSCARPA m'a aidée à constater comment ma mère adapte sa conduite automobile selon les difficultés qu'il expérimente. » [P13]. Par ailleurs, l'OSCARPA a aidé les proches à mieux connaître les signes d'une conduite automobile moins sécuritaire (ex: contraventions, accidents, etc.), tel que rapporté par un des participants : "J'ai apprécié les questions et les diagrammes de l'OSCARPA, puisque cela m'a aidé à situer ma mère par rapport à la conduite automobile et à statuer si elle est sécuritaire. » [P40]. Ce participant a réalisé grâce à la lecture de l'outil que, lorsque des doutes surgissent en lien avec la sécurité du conducteur, plusieurs solutions peuvent être mises en place avant de considérer l'arrêt de la conduite. L'OSCARPA leur a aussi permis de constater les stratégies utilisées par le conducteur âgé afin d'adapter sa conduite automobile et, à l'aide de l'arbre décisionnel, d'avoir une meilleure idée de ce qu'ils doivent considérer lorsqu'ils s'intéressent à la conduite automobile d'un aîné. Enfin, lors des rencontres individuelles, un grand nombre de participants ont mentionné que l'OSCARPA était un bon outil pour faciliter la communication, mais que la conduite automobile demeurait un sujet difficile à aborder avec un conducteur âgé. Certains participants rapportent quelques améliorations possibles. En effet, un participant rapporte que : "Selon le document [( l'OSCARPA), le nouveau moyen de transport] serait un taxi (trop cher). Mes parents demeurent en campagne (des solutions) ?" [P33]. Ainsi, une prochaine version de l'OSCARPA pourrait considérer davantage les ressources disponibles en milieu rural. Deux participants [P14 et P17] rapportent aussi que l'outil serait davantage utile pour

Tableau 4 : Résultats du questionnaire Changements des Habiletés pour la conduite Automobile perçus par les Proches d'Aînés $($ CHAPA; $n=45$ )

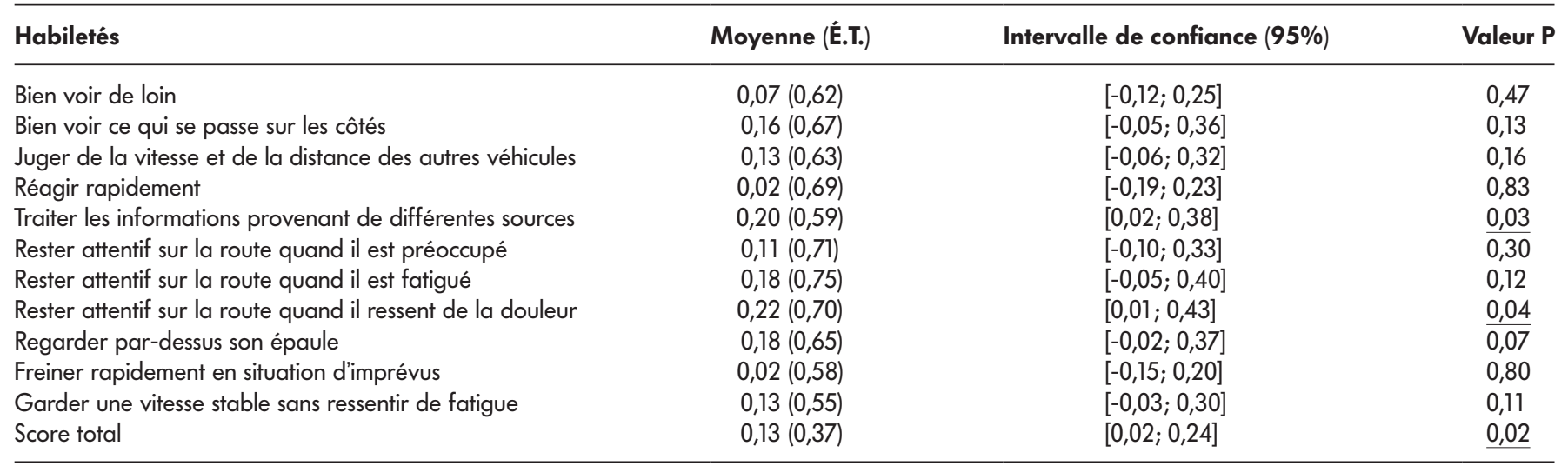


Tableau 5 : Résultats pour le questionnaire Utilisation de Stratégies Compensatoires perçue par les Proches d'Aînés(USCPA)

\begin{tabular}{|c|c|c|c|}
\hline Stratégies compensatoires & Moyenne (É.T.) & Intervalle de confiance (95\%) & Valeur $\mathbf{P}$ \\
\hline Éviter de conduire à la noirceur & $0(0,52)$ & {$[-0,16 ; 0,16]$} & 1,00 \\
\hline Être plus vigilant aux intersections & $0,18(0,68)$ & {$[-0,03 ; 0,38]$} & 0,09 \\
\hline Éviter les situations de virage à gauche & $0,13(0,55)$ & {$[-0,03 ; 0,30]$} & 0,11 \\
\hline Éviter les autoroutes achalandées & $0,04(0,64)$ & {$[-0,15 ; 0,24]$} & 0,64 \\
\hline Être plus conscient de ce qui se passe tout autour de lui & $0,24(0,57)$ & {$[0,07 ; 0,42]$} & $\leq 0,01$ \\
\hline Faire de l'exercice physique & $0,13(0,59)$ & {$[-0,04 ; 0,31]$} & 0,14 \\
\hline $\begin{array}{l}\text { Porter une attention particulière à la circulation, aux panneaux } \\
\text { de signalisation, aux feux de circulation, aux cyclistes } \\
\text { et aux piétons à l'approche des intersections }\end{array}$ & $0,22(0,60)$ & {$[0,04 ; 0,40]$} & $\underline{0,02}$ \\
\hline Planifier le trajet à l'avance & $0,07(0,75)$ & {$[-0,16 ; 0,29]$} & 0,55 \\
\hline Installer des miroirs spéciaux sur le véhicule & $0,07(0,39)$ & {$[-0,05 ; 0,19]$} & 0,26 \\
\hline Choisir des routes qui requièrent peu de changements de voie & $0,18(0,65)$ & {$[-0,02 ; 0,37]$} & 0,07 \\
\hline Suivre un programme d'exercices pour améliorer sa force et sa flexibilité & $0,02(0,58)$ & {$[-0,15 ; 0,20]$} & 0,80 \\
\hline Conduire à la limite de vitesse permise & $0,20(0,59)$ & {$[0,02 ; 0,38]$} & 0,03 \\
\hline $\begin{array}{l}\text { Se questionner sur la cause des réactions négatives des autres } \\
\text { automobilistes à son égard }\end{array}$ & $0,16(0,60)$ & {$[-0,03 ; 0,34]$} & $\overline{0,09}$ \\
\hline Être à l'écoute des commentaires de son entourage concernant sa conduite & $0,24(0,68)$ & {$[0,04 ; 0,45]$} & 0,02 \\
\hline Score total & $0,14(0,26)$ & {$[0,06 ; 0,22]$} & $\overline{0,001}$ \\
\hline
\end{tabular}

des conducteurs âgés de plus de 65 ans qui nécessitent des discussions et des actions en lien avec une diminution de leurs capacités ou avec l'arrêt de la conduite.

\section{Discussion}

Cette étude visait à explorer les effets de l'OSCARPA sur i) l'intérêt, l'ouverture et les connaissances des proches, et leur perception sur ii) les changements des habiletés d'un conducteur âgé et iii) l'utilisation des stratégies compensatoires. À la suite de la lecture de l'OSCARPA et de l'utilisation du journal de bord, les proches présentent un intérêt, une ouverture et des connaissances accrus sur la conduite automobile, ainsi $\mathrm{qu}^{\prime}$ 'une amélioration modeste de leur perception des changements des habiletés et de l'utilisation de stratégies compensatoires par un conducteur âgé de leur entourage. Ces résultats sont similaires à ceux d'autres études réalisées auprès de conducteurs âgés qui ont démontré les bénéfices d'interventions éducationnelles sur la conduite automobile. Malgré le fait qu'elles ne portaient pas sur la même clientèle, ces études représentent, en l'absence de recherche sur les proches de conducteurs âgés, le meilleur comparatif possible. Par exemple, selon Owsley, Stalvey et Phillips (2003) et comparativement à ceux n'ayant pas reçu l'intervention, les conducteurs ayant participé à un groupe éducationnel sur les problèmes visuels et l'autorégulation, utiliseraient davantage des stratégies compensatoires. De plus, à la suite de l'OSCAR, l'intérêt, l'ouverture et les connaissances des conducteurs âgés se sont améliorés (Levasseur et al., 2014 ; Levasseur et al., 2015), tel que nous l'avons observé dans la présente étude chez les proches. Plus spécifiquement et comparativement aux conducteurs âgés ayant participé à l'OSCAR, la présente étude a permis une augmentation supérieure des connaissances des proches sur les effets du vieillissement, sur les stratégies compensatoires, sur la sécurité routière et sur les habiletés requises. Puisqu'ils sont moins directement impliqués dans la conduite de l'aîné, les proches pourraient ainsi présenter des habiletés supérieures à l'amélioration de leurs connaissances sur le sujet.

Par ailleurs, les résultats obtenus démontrent que l'OSCARPA favorise globalement la prise de conscience des proches sur les changements des habiletés et l'utilisation des stratégies compensatoires. Ces résultats soutiennent qu'il est possible, grâce à une intervention simple, de modifier les perceptions des proches en lien avec les habiletés d'un conducteur âgé et son utilisation des stratégies compensatoires. Plus spécifiquement, des changements ont été rapportés en lien avec deux habiletés et cinq stratégies compensatoires. L'absence de changement pour certaines habiletés (ex. habileté à bien voir de loin) et quelques stratégies compensatoires (ex. éviter de conduire à la noirceur) peut s'expliquer 
par le fait que les proches étaient, même avant l'OSCARPA, sensibilisés à la condition du conducteur âgé ou à son utilisation de stratégies.

Cette amélioration de la prise de conscience demeure tout de même modeste et peut être expliquée par la complexité des changements de comportements (Prochaska \& Velicer, 1997), dont l'utilisation de nouvelles stratégies compensatoires par un conducteur âgé fait partie. En effet, avant d'utiliser ces stratégies, le conducteur âgé doit d'abord de reconnaître que ses habiletés ont diminué et doit ensuite planifier de les utiliser. Également, l'outil n'était pas personnalisé au conducteur âgé, ne l'impliquait pas directement et devait être utilisé par le proche dans un lapse de temps relativement court (8 à 10 semaines). Une étude a d'ailleurs démontré que les interventions plus intensives étaient plus efficaces (Jones et al., 2012).

La sensibilisation sur la conduite automobile demeure ainsi complexe et il est difficile pour les proches de mentionner à un conducteur âgé qu'il est temps d'arrêter de conduire (Aging Parents and Elder Care, 2009) ou d'utiliser des stratégies compensatoires. Une récente étude a d'ailleurs démontré que la majorité des proches anticipaient une réaction négative en lien avec la discussion sur la conduite automobile (Frost-Steward, 2015). La réticence des proches à intervenir est d'ailleurs une des barrières à l'utilisation de stratégies compensatoires par les conducteurs âgés (Levasseur, Coallier, Bédard, Beaudry, Arcand, Langlais et Therrien, 2015). Aussi, pour convaincre un conducteur âgé de cesser de conduire, il est souhaitable et essentiel qu'un proche en discute avec lui (SAAQ, 2011). Un professionnel de la santé peut aussi, au besoin, faciliter cette discussion. Afin de diminuer la colère engendrée par la cessation, il est cependant primordial d'inclure le conducteur âgé dans les discussions (Senior Solutions of America, 2007).

\section{Forces et limites}

Cette étude a permis le développement et une première validation de l'OSCARPA, une intervention de sensibilisation des proches à une conduite automobile sécuritaire de conducteurs âgés. Jusqu'à maintenant, il n'existait pas d'outil complet pour les proches ayant fait l'objet d'une validation. Compte tenu de son caractère exploratoire, cette étude a aussi des limites. D'abord, elle a été réalisée avec un échantillon de convenance dont l'intérêt, l'ouverture et les connaissances des participants envers la conduite automobile peuvent avoir été supérieurs. Afin d'éviter une surestimation des effets, une exploration optimale des changements par les questionnaires était difficile. Ainsi, les changements pourraient avoir été sous-estimés. Par exemple, un proche qui rapportait à $\mathrm{T}_{0}$ " aucun " problème en lien avec la vision de l'aîné et qui, à la suite d'une dis- cussion avec l'aîné, répondait à $\mathrm{T}_{1}$ " je ne sais pas », présentait un changement négatif puisqu'il avait maintenant des doutes. Ces doutes peuvent toutefois représenter une sensibilisation et une reconnaissance accrues du proche en lien avec les difficultés du conducteur âgé et peuvent engendrer un intérêt accru. Les études sur l'évaluation de l'OSCARPA doivent se poursuivre à l'aide d'un essai clinique à répartition aléatoire incluant un groupe contrôle actif, c.-à-d recevant une autre intervention, pour vérifier si les effets obtenus sont attribuables à l'intervention et non à l'évaluation. Les études doivent aussi explorer les effets sur une plus longue période de temps pour permettre l'observation des habiletés ou l'utilisation des stratégies par le conducteur âgé. Enfin, l'inclusion du conducteur âgé dans ces études permettrait de favoriser les discussions et de comparer les perceptions du proche avec celles de l'aîné ou même avec des observations objectives des changements et des stratégies utilisées par ce dernier.

\section{Implications cliniques}

L'OSCARPA peut permettre de sensibiliser et de soutenir les proches dans leur rôle en lien avec une conduite automobile sécuritaire des aînés. Tel que rapporté par les participants de la présente étude, l'outil permet de faciliter la discussion entre le proche et l'aîné sur la conduite automobile, un sujet tabou. Les ressources identifiées dans l'OSCARPA permettent également aux proches de mieux connaître les services qui peuvent les soutenir en lien avec les besoins d'un conducteur âgé. En augmentant l'intérêt, l'ouverture et les connaissances des proches d'un conducteur âgé, les professionnels de la santé auront d'importants alliés pour favoriser une conduite automobile sécuritaire et responsable.

\section{Conclusion}

Cette étude a permis de démontrer que l'OSCARPA favorise une augmentation de l'intérêt, de l'ouverture et des connaissances des proches d'un conducteur âgé. De plus, malgré un changement plus modeste de leurs perceptions, cette intervention peut informer les proches sur les habiletés requises pour une conduite automobile sécuritaire des aînés et sur les stratégies compensatoires qu'ils peuvent utiliser. Les prochaines études doivent vérifier si l'OSCARPA permet aux proches de mieux soutenir les conducteurs âgés lors des changements qui surviennent avec le vieillissement et de les assister dans l'utilisation de stratégies compensatoires. D'autres études sont aussi nécessaires afin de mieux connaître les effets de l'utilisation de l'OSCARPA en clinique et les besoins des proches en lien avec la discussion sur la conduite automobile et l'accompagnement à l'utilisation de stratégies. Ces études pourraient aussi explorer l'intensité requise et la possibilité de personnaliser l'intervention à l'aîné. Par ailleurs, puisqu'une étude a démontré que les perceptions 
sont liées aux habiletés à réguler ses comportements (Blanchard \& Myers, 2010), il serait intéressant d'approfondir les effets des conseils de l'OSCARPA sur la perception des habiletés, l'autorégulation et l'estime de soi des conducteurs âgés. D'autres études pourraient aussi porter sur la combinaison de l'OSCARPA avec d'autres interventions, par exemple, avec le programme éducatif Carfit (Craik, Stern, Lapointe, McCarthy, \& Schold Davis, 2015) qui offre aux conducteurs âgés une vérification rapide et complète de leur véhicule selon leurs besoins. Aussi, afin de renouveler leurs compétences, le programme 55 ans au volant (Conseil canadien de la sécurité, 2007) offre aux personnes âgées de 50 ans et plus une mise à jour. Il pourrait d'ailleurs être intéressant pour les proches de participer à ce programme en même temps que le conducteur âgé. Enfin, si l'OSCAR et l'OSCARPA sont ultimement implantés à grande échelle dans la communauté, il sera possible de vérifier si ces deux outils permettent de prévenir les accidents et les blessures des conducteurs âgés, tout en maintenant leur participation sociale dans la communauté, ainsi que leur estime de soi.

\section{Références}

ACE. (2013). Restez sur la bonne voie : Conduisez prudemment tout en prenant de l'âge. Disponible sur : http:/ / www. olderdriversafety.ca/consumer/resources/documents / Families_FR.pdf. Accédé le 15 septembre 2013.

Aging Parents and Elder Care: Helping People Overcome the Challenges of Elder Care. (2009). Checklist: When to Put the Brakes on Elderly Drivers. Disponible sur : http:/ / www.aging-parents-and-elder-care.com/Pages / Checklists/Elderly_Drivers.html. Accédé le 8 avril 2015.

Association canadienne des ergothérapeutes (ACE). (2009). Les conducteurs âgés au Canada et leur famille. Plan d'action national pour la prévention des blessures chez les conducteurs âgés. Disponible sur : http://www.securitedesconducteursages.ca/consommateur/index. html. Accédé le 14 août 2013.

Audet, T., Arcand, M., Godbout, C., et Lessard, L. (2007). Conduite automobile. Dans: M. Arcand \& R. Hébert. Précis pratique de gériatrie, 3e éd. (pp. 1099-1112). Edisem, Saint-Hyacinte, Québec, Canada.

Association canadienne des automobilistes (CAA). (2011). Guide pratique : Comment aider un aîné au volant pour des déplacements sécuritaires. Disponible sur : https:/ /www. caaquebec.com/fileadmin/...la.../GuidePratiqueAines2. pdf. Accédé le 14 août 2013.

Blanchard, R. A., \& Myers, A. M. (2010). Examination of driving comfort and self-regulatory practices in older adults using in-vehicle devices to assess natural driving patterns. Accident Analysis \& Prevention, 42, 1213-1219.

Club de l'assurance automobile (CAA), (2011), Comment aider un aîné au volant pour des déplacements sécuritaires, Disponible sur: https://www.caaquebec.com/fileadmin/ documents / PDF/Sur_la_route/Securite_routiere / GuidePratiqueAines2.pdf, Accédé le 30 avril 2017

Classen, S., Winter, S. M., Velozo, C. A., Bédard, M., Lanford, D. N., Brumback, B., et al. (2010). Item development and validity testing for a self- and proxy report: the safe driving behavior measure. American Journal of Occupational Therapy, 64, 296-305.

Craik, J., Stern, E., Lapointe, J., McCarthy, N., \& Schold Davis, E. (2015). "CarFit Canada: Lessons learned from an international collaboration." World Federation of Occupational Therapists, Bulletin, 71(1), 22-25.

Crepeau, E. B., Cohn, E. S., Schell, B. A. B., \& Willard, H. S. (2009). Willard E Spackman's Occupational Therapy. $11^{\text {th }}$ ed. Baltimore, MD, États-Unis: Wolters Kluwer Health/ Lippincott Williams \& Wilkins.

Conseil canadien de la sécurité. (2007). Dépliant 55 ans au volant, 5e éd. [Brochure].

D’Ambrosio, L., Coughlin, J. F., Mohyde, M., Carruth, A., Hunter, J. C., \& Stern, R. A. (2009). Caregiver communications and the transition from driver to passenger among people with dementia. Topics in Geriatric Rehabilitation, 25, 33-42.

Eberhard, J. W., Stutts, J., Burkhardt, J., Finn, J., Hunt, L., Staplin, L., et al. (2006). Strategies and tools to enable safe mobility for older adults. Topics in Geriatric Rehabilitation, 22(1), 3-17.

Eby, D. W., \& Molnar, L. J. (2010). M-CASTL 2010 synthesis report - Volume 1: Older adult safety and mobility. University of Michigan: Ann Arbor.

Friedland, J., \& Rudman, D. L. (2009). From Confrontation to Collaboration: Making a Place for Dialogue on Seniors' Driving. Topics in Geriatric Rehabilitation, 25, 12-23.

Fougeyrollas, P. (2010). Le funambule, le fil et la toile. Transformations réciproques du sens du handicap. Québec, QC: Presses de l'Université Laval.

Frost-Steward, J. M. (2015). It seems like it should be so simple: The role of the family in elder driving retirement [Dissertation doctorale]. Manhattan, KS, États-Unis: Université du Kansas.

Jones, V., Gielen, A., Bailey, M., Rebok, G., Agness, C., Soderstrom, C., et al. (2012). The effect of a low and high resource intervention on older drivers' knowledge, behaviors and risky driving. Accident Analysis E Prevention, 49, 486-492.

Joseph, C. B. (2010). Physician's Guide to Assessing and Counseling Older Drivers, 2d ed. Chicago, IL: American Medical Association.

King, M. L. (2009). How a caregiver stops an unsafe elderly driver. Disponible sur : https://suite.io/mary-king/2n2e25t. Accédé le 29 septembre 2013.

Kostyniuk, L. P., \& Molnar, L. J. (2008). Self-regulatory driving practices among older adults: Health, age and sex effects. Accident Analysis \& Prevention, 40, 1576-80. 
Levasseur, M., Audet, T., Gelinas, I., Bedard, M., Langlais, M. È., Therrien, F. H., et al. (2015). Awareness tool for safe and responsible driving (OSCAR): A potential educational intervention for increasing interest, openness and knowledge about the abilities required and compensatory strategies among older drivers. Traffic injury prevention, 16(6), 578-586.

Levasseur, M., Audet, T., Gélinas, I., Bédard, M., Renaud, J., Coallier, J. C., et al. (2014). Outil de sensibilisation des conducteurs âgés aux capacités requises pour une conduite automobile sécuritaire et responsable (OSCAR): développement et validation. Recherche Transports Sécurité, 2014(04), 257-269.

Levasseur, M, Coallier, J. C., Gabaude, C., Beaudry, M., Bédard, M., Langlais, M. E., et al. (2016). Facilitators, barriers and needs in the use of adaptive driving strategies to enhance older drivers' mobility: Importance of openness, perceptions, knowledge and support. Transportation Research Part F: Traffic Psychology and Behaviour, 56-66.

Liddle, J., \& McKenna, K. (2003). Older drivers and driving cessation. The British Journal of Occupational Therapy, 66(3), 125-32.

Michon, J. A. (1985). A critical view of driver behavior models: What do we know, what should we do? In Human behavior and traffic safety (pp. 485-524). Springer US.
Owsley, C., Stalvey, B. T., \& Phillips, J. M. (2003). The efficacy of an educational intervention in promoting self-regulation among high-risk older drivers. Accident Analysis E Prevention, 35, 393-400.

Prochaska, J., \& Velicer, W. (1997). The transtheorical model of health behavior change. American Journal of Health Promotion, 12, 38-48.

Sedgwick County Department on Aging. (2010). We need to talk - Family conversations with older drivers. Disponiblesur:http: / /hartfordauto.thehartford.com/ UI/Downloads/FamConHtd.pdf. Accédé le 14 août 2013.

Senior solutions of America. (2007). Elderly drivers: Is your loved one driving safely? Disponible sur : http:// elderlydrivingassessments.com/images/individ/ elderdriversfamilychecklist.pdf. Accédé le 1er août 2013

Société de l'assurance automobile du Québec (SAAQ). (2011). Au volant de ma santé. Disponible sur: www.saaq.gouv. qc.ca/publications/prevention/volant_sante.pdf. Accédé le 14 août 2013.

Turcotte, M. (2012). Profil des habitudes liées au transport chez les aînés. Disponible sur: http:/ / www.statcan.gc. ca/pub/11-008-x/2012001/article/11619-fra.htm\#a2/. Accédé le 12 novembre 2014. 


\section{Annexes}

Annexe 1 : Interprétation des changements entre $T_{0}$ et $T_{1}$ au questionnaire CHAPA

\begin{tabular}{|c|c|c|c|c|}
\hline $\begin{array}{l}\text { Choix de } \\
\text { réponse à }\end{array}$ & Observé & Rapporté & Aucun & Je ne sais pas \\
$\begin{array}{l}\text { Choix } \\
\text { de } \\
\text { réponse } \\
\text { à } \mathrm{T}_{0}\end{array}$ & & & & \\
\hline Observé & 0 & -1 & -1 & -1 \\
\hline Rapporté & 1 & 0 & -1 & -1 \\
\hline Aucun & 1 & 1 & 0 & 0 \\
\hline Ne sait pas & 1 & 1 & 1 & -1 \\
\hline
\end{tabular}

Annexe 2: Interprétation des changements entre $T_{0}$ et $T_{1}$ au questionnaire USCPA

\begin{tabular}{|c|c|c|c|c|}
\hline $\begin{array}{l}\text { Choix de } \\
\text { réponse à } \\
\mathrm{T}_{1}\end{array}$ & Observé & Rapporté & Non utilisée & Je ne sais pas \\
$\begin{array}{l}\text { Choix } \\
\text { de } \\
\text { réponse } \\
\text { à } \mathrm{T}_{0}\end{array}$ & & & & \\
\hline Observé & 0 & -1 & -1 & -1 \\
\hline Rapporté & 1 & 0 & -1 & -1 \\
\hline Non utilisé & 1 & 1 & 0 & -1 \\
\hline Ne sait pas & 1 & 1 & 1 & 0 \\
\hline
\end{tabular}


Annexe 3 : Total des changements pré et post test pour chaque habileté au questionnaire CHAPA.

CHAPA Post-test

\begin{tabular}{|c|c|c|c|c|c|c|c|}
\hline $\begin{array}{c}\text { Changement } \\
\text { observé }\end{array}$ & $\begin{array}{l}\text { Changement } \\
\text { rapporté }\end{array}$ & $\begin{array}{c}\text { Aucun } \\
\text { Changement }\end{array}$ & $\begin{array}{c}\text { Je ne } \\
\text { sais pas }\end{array}$ & $\begin{array}{c}\text { Changement } \\
\text { observé }\end{array}$ & $\begin{array}{c}\text { Changement } \\
\text { rapporté }\end{array}$ & $\begin{array}{c}\text { Aucun } \\
\text { Changement }\end{array}$ & $\begin{array}{c}\text { Je ne } \\
\text { sais pas }\end{array}$ \\
\hline 5 & 6 & 29 & 6 & 6 & 4 & 32 & 4 \\
\hline 8 & 4 & 22 & 11 & 9 & 2 & 30 & 4 \\
\hline 7 & 3 & 24 & 11 & 9 & 2 & 30 & 4 \\
\hline 9 & 4 & 23 & 9 & 10 & 2 & 27 & 6 \\
\hline 7 & 1 & 26 & 11 & 11 & 5 & 27 & 4 \\
\hline 8 & 1 & 21 & 14 & 8 & 5 & 26 & 6 \\
\hline 4 & 6 & 16 & 19 & 6 & 7 & 23 & 9 \\
\hline 2 & 3 & 16 & 24 & 5 & 4 & 24 & 13 \\
\hline 9 & 3 & 23 & 10 & 12 & 2 & 27 & 4 \\
\hline 4 & 2 & 29 & 10 & 6 & 0 & 32 & 7 \\
\hline 3 & 2 & 28 & 12 & 6 & 4 & 29 & 6 \\
\hline 66 & 35 & 257 & 137 & 88 & 37 & 307 & 67 \\
\hline
\end{tabular}


Annexe 4 : Total des changements pré- et post-test pour chaque stratégie au questionnaire USCPA.

USCPA Pré-test

USCPA Post-test

\begin{tabular}{lllllllll}
$\begin{array}{l}\text { Stratégie } \\
\text { observée }\end{array}$ & $\begin{array}{c}\text { Stratégie } \\
\text { rapportée }\end{array}$ & $\begin{array}{c}\text { Stratégie } \\
\text { non utilisée }\end{array}$ & $\begin{array}{c}\text { Je ne } \\
\text { sais pas }\end{array}$ & $\begin{array}{c}\text { Stratégie } \\
\text { observée }\end{array}$ & $\begin{array}{c}\text { Stratégie } \\
\text { rapportée }\end{array}$ & $\begin{array}{c}\text { Stratégie } \\
\text { non utilisée }\end{array}$ & $\begin{array}{c}\text { Je ne } \\
\text { sais pas }\end{array}$ \\
\hline
\end{tabular}

Éviter de conduire à la noirceur

Être plus vigilant aux intersections

Regarder à droite de la chaussée plutôt que les phares des voitures

venant en sens inverse

Éviter les situations de virage à gauche

Éviter les autoroutes achalandées

Éviter les heures de pointe

Laisser une plus grande distance entre le véhicule et celui à l'avant

Être plus conscient de ce qui se passe tout autour de lui

Faire de l'exercice physique

Porter une attention particulière à la circulation, aux panneaux de

signalisation $[\ldots]$ intersections

Planifier le trajet à l'avance

Planifier l'achat d'une voiture à transmission automatique

Éviter de conduire lors de conditions pouvant interférer avec

l'habileté à se concentrer

Réduire les distractions au volant

Installer des miroirs spéciaux sur le véhicule

Choisir des routes qui requièrent peu de changements de voie

Suivre un programme d'exercices pour améliorer sa force et flexibilité

Conduire à la limite de vitesse permise

Se questionner sur la cause des réactions négatives des autres automobilistes à son égard

Être à l'écoute des commentaires de son entourage concernant sa conduite

\begin{tabular}{|c|c|c|c|c|c|c|c|}
\hline 19 & 15 & 11 & 4 & 17 & 17 & 10 & 4 \\
\hline 11 & 5 & 16 & 14 & 16 & 8 & 16 & 6 \\
\hline 1 & 2 & 16 & 26 & 8 & 3 & 21 & 13 \\
\hline 3 & 2 & 29 & 12 & 4 & 6 & 27 & 10 \\
\hline 11 & 7 & 20 & 7 & 12 & 10 & 20 & 4 \\
\hline 11 & 9 & 18 & 8 & 14 & 11 & 17 & 4 \\
\hline 11 & 5 & 20 & 11 & 18 & 8 & 14 & 7 \\
\hline 8 & 1 & 19 & 17 & 15 & 4 & 18 & 9 \\
\hline 12 & 7 & 21 & 6 & 14 & 8 & 20 & 3 \\
\hline 8 & 4 & 18 & 16 & 15 & 7 & 15 & 11 \\
\hline 17 & 12 & 11 & 7 & 16 & 16 & 13 & 2 \\
\hline 8 & 7 & 29 & 4 & 11 & 5 & 29 & 2 \\
\hline 14 & 9 & 16 & 7 & 12 & 17 & 11 & 6 \\
\hline 12 & 4 & 22 & 9 & 10 & 11 & 22 & 4 \\
\hline 7 & 1 & 34 & 3 & 6 & 1 & 37 & 1 \\
\hline 3 & 3 & 29 & 10 & 7 & 8 & 25 & 7 \\
\hline 7 & 4 & 31 & 4 & 7 & 5 & 29 & 4 \\
\hline 16 & 7 & 16 & 8 & 18 & 10 & 15 & 3 \\
\hline 5 & 3 & 21 & 17 & 6 & 4 & 23 & 12 \\
\hline 7 & 3 & 22 & 13 & 17 & 6 & 17 & 7 \\
\hline
\end{tabular}


Copyright of Canadian Journal on Aging is the property of Cambridge University Press and its content may not be copied or emailed to multiple sites or posted to a listserv without the copyright holder's express written permission. However, users may print, download, or email articles for individual use. 